\title{
Research on Air Noise Measurement of Marine Equipment and Its Uncertainty
}

\author{
Yongchao Cheng ${ }^{*}$, Chengming Dai, Miao Zhang, Yaofei Li
}

The Second Ship Design Institute of Wuhan, Wuhan, China

Email address:

15997433862@126.com (Yongchao Cheng)

${ }^{*}$ Corresponding author

\section{To cite this article:}

Yongchao Cheng, Chengming Dai, Miao Zhang, Yaofei Li. Research on Air Noise Measurement of Marine Equipment and Its Uncertainty. American Journal of Mechanical and Industrial Engineering. Vol. 6, No. 5, 2021, pp. 77-80. doi: 10.11648/j.ajmie.20210605.14

Received: July 16, 2021; Accepted: October 25, 2021; Published: November 5, 2021

\begin{abstract}
Uncertainty measurement is one of the important indexes to quantitatively describe the quality of measurement results, which directly reflect the reliability, feasibility and comparability of the measurement results, meanwhile, keep the noise measured under the requirement of quality control. In this paper, By designing the air noise test of ship air compressor and scientifically calculating the uncertainty of the test, Starting from many factors affecting the vibration and noise test, and considering all factors affecting the test results, the scientific analysis system of uncertainty is used. A good reference basis is provided for the uncertainty calculation of subsequent tests. firstly measure the air noise of marine equipment based on GJB 4058-2000, then according to JJF 1059-2012, analyze and evaluate the uncertainty of measurement in detail: analyzing the sources of measurement uncertainty as well as giving the results of measurement uncertainty. scientific protection of sound level meter and other precision equipment should be considered especially. The environment in which the sound level meter is placed has a great influence on the sensitivity of the sound level meter, so the effectiveness of measurement can be improved by placing the sound level meter in the incubator after the measurement verification. This research is suitable for practical application.
\end{abstract}

Keywords: Air Noise, Measurement, Measurement Uncertainty, Analysis and Evaluation

\section{Introduction}

Usually the quality of the measurement result is measured by the measurement error, but the measurement error can only show the short-term quality of the measurement. Whether the measurement process is under continuous control, whether the measurement results can remain stable and consistent, and whether the measurement capability meets the requirements of production profitability, it needs to be measured by measurement uncertainty. The greater the measurement uncertainty, the worse the measurement ability; on the contrary, the stronger the measurement ability. However, no matter how small the measurement uncertainty is, the measurement uncertainty range must include the true value (generally replaced by the agreed true value), otherwise the measurement process has failed.

The purpose of measurement is to determine the measured value. The quality of the measurement results is the most important basis for measuring the credibility of the measurement results. Measurement uncertainty is a quantitative characterization of the quality of measurement results, and the availability of measurement results largely depends on the size of its uncertainty. Therefore, the measurement result statement must include the value assigned to the measured value and the measurement uncertainty associated with the value at the same time, to be complete and meaningful.

Using noise detector to measure the air noise is an important means in the control of vibration attenuation and noise reduction. In this process, instruments used, measuring method and measurement procedure always keep invariant, and similarity as measuring objects. Generally measurement process is under some verification, calibration specification and national standard, however, measurements differ each other. In order to keep every measurement scientific and accurate, uncertainty concept has to be introduced. [1]

Uncertainty measurement is one of the important indexes to quantitatively describe the quality of measurement results, which directly reflect the reliability, feasibility and comparability of the measurement results, meanwhile, keep 
the noise measured under the requirement of quality control. Only combined with its uncertainty does noise measurement make sense.

In this paper, the air noise of marine air compressor is measured. Then analyze the sources of measurement uncertainty and evaluate it comprehensively. Through the above, summarize the general method of evaluating marine equipments air noise measurement uncertainty, and this method has strong engineering practical significance.

\section{Air Measurement of Marine Equipment}

The test object is marine air compressor, whose basic parameters as follows: power $(7.5 \mathrm{~kW})$, displacement $(1.4$ $\left.\mathrm{m}^{3} / \mathrm{min}\right)$, exhaust pressure $(0.7 \mathrm{MPa})$, speed $(850 \mathrm{rpm})$, mass $(335 \mathrm{~kg})$, boundary dimension $(1690 \times 530 \times 1260 \mathrm{~mm})$. The outline diagram is as shown as figure 1.

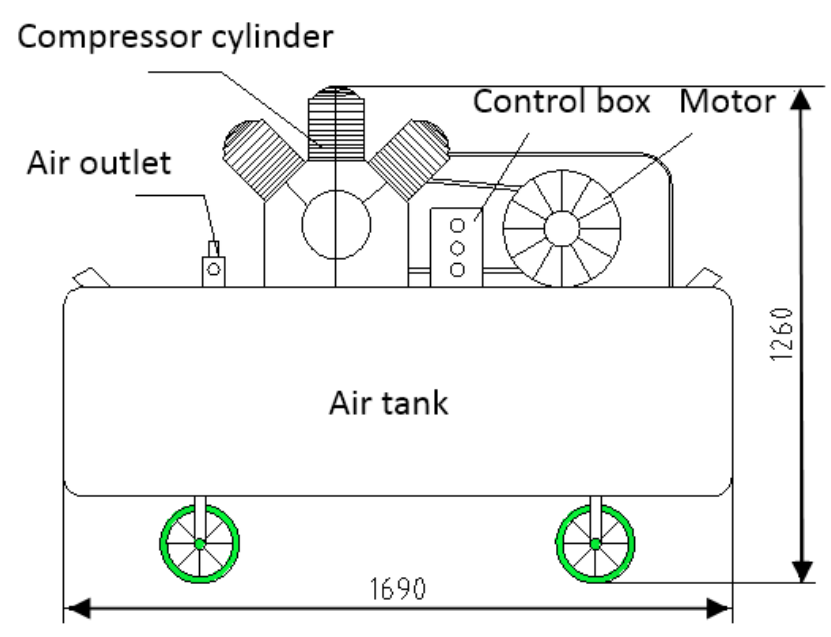

Figure 1. Outline diagram of test object.

The marine air compressor air noise is tested based on the method in GJB 4058-2000 《measurement method of noise and vibration of marine equipment》 [2]. In order to reduce systematical error, 5 sets of measured data was recorded [3].

The noise instrument used in this air compressor noise test is the sound level meter produced by B\&K (Denmark), whose transducer type is 2236 . The calibrator is acoustic calibrator by B\&K with type 4189 .

\section{Instrument preparation}

Install the calibrator on the sound level meter weight to A sound pressure level, and adjust the reading to be $94 \mathrm{~dB}$. Then uninstall the calibrator after calibration.

Air noise test

Air noise measurement of marine equipment adopts A sound level and fast measurement mode. One reading per second, one minute. Finally LeqA is provided.

\section{Test check-up}

Recalibrate the sound level meter after testing, the sound level meter reading shall be $94 \mathrm{~dB}$, otherwise, the measurement should be checked even redo.
Analyze the test method and environmental factors, the sources of uncertainty in this measurement are as follows:

1) Uncertainty contribution from repeatability test;

2) Uncertainty contribution from sound level meter calibration;

3) Uncertainty contribution from sound level meter definition;

4) Uncertainty contribution from test radius error;

5) Uncertainty contribution from test environment reflection;

6) Uncertainty contribution from temperature, humidity, pressure.

From the above, Uncertainty contribution from repeatability measurement can be evaluated by A class evaluation of standard uncertainty, the other five can be evaluated by B class evaluation of standard uncertainty. [4]

\section{Measurement Uncertainty Evaluation}

Because of the measurement error, measurement uncertainty defines the degree of uncertainty within a certain range of the true value to be measured. That is to say, the smaller the measurement uncertainty, the better.

A class evaluation of standard uncertainty

A class evaluation of standard uncertainty is the best estimation of the error of existing test data by statistical analysis method.

There is no need to modify the test data, for tester is same and background noise of every measuring point is $10 \mathrm{~dB}$ lower than measured value. Measuring points diagram is shown as figure 2 and test data is shown as table 1.
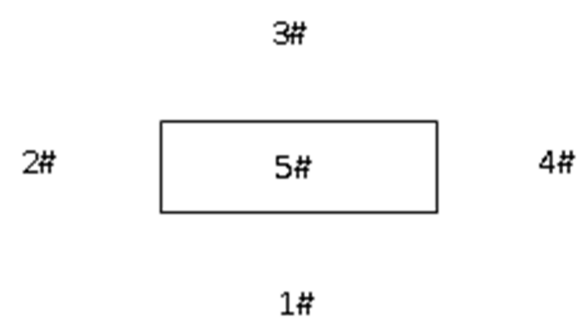

Figure 2. Measurement point drawing.

Table 1. Air noise test data of air compressor.

\begin{tabular}{llllll}
\hline \multirow{2}{*}{$\begin{array}{l}\text { Test } \\
\text { number }\end{array}$} & \multicolumn{6}{l}{ Measurement results dB (A) } \\
\cline { 2 - 6 } & $\mathbf{1 \#}$ & $\mathbf{2 \#}$ & $\mathbf{3 \#}$ & 4\# & $\mathbf{5 \#}$ \\
\hline 1 & 93.1 & 89.3 & 91.6 & 90.9 & 94.0 \\
2 & 92.6 & 90.0 & 91.2 & 90.3 & 93.7 \\
3 & 92.9 & 89.7 & 91.8 & 90.7 & 93.9 \\
4 & 93.3 & 89.4 & 92.2 & 90.9 & 94.2 \\
5 & 92.8 & 89.8 & 91.4 & 90.5 & 93.6 \\
\hline
\end{tabular}

Test standard deviation from Bessel formula $\mathrm{s}(\mathrm{x})=0.29$ $\mathrm{dB}(\mathrm{A})$, thus uncertainty component caused by the repeatability of noise measurement is $\mathcal{U}_{A}=\frac{s(x)}{\sqrt{n}}=0.13 \mathrm{~dB}(A)$, its degree of freedom is $\boldsymbol{V}_{A}=5-1=4$. 


\section{B Class Evaluation of Standard Uncertainty}

B class evaluation of standard uncertainty refers to the uncertainty that does not conform to the statistical law, which is mainly caused by the accuracy limit of the instrument and equipment itself and other system factors.

1) Uncertainty contribution from sound level meter calibration

From the calibration test report of sound level meter [5-14], we can find that: the expanded uncertainty $U_{95}=0.19 \mathrm{~dB}$, confidence level $\mathrm{p}=95 \%$. Uncertainty reliability given by calibration report is taken as $90 \%$, according to JJF1059.1-2012, degree of freedom is taken as 50, $k=t_{p}=2.01$, thus we can conclude that the standard uncertainty considering calibration is $\mathcal{U}_{B 1}=\frac{U}{k}=\frac{0.19}{2.01}=0.095 \mathrm{~dB}(A)$.

2) Uncertainty contribution from sound level meter definition

The resolution $\mathrm{j}$ of 2236 instrument display screen is an important factor to produce measurement uncertainty. Its standard deviation is $\mathrm{u}=0.29 \mathrm{j}$. The $\mathrm{LCD}$ resolution of 2236 sound level meter is $0.1 \mathrm{~dB}$, therefore uncertainty contribution from sound level meter definition is $\boldsymbol{U}_{B 2}=0.29 * 0.1=0.029 \mathrm{~dB}(A)$. The resolution of digital instrument display is relatively intuitive, so it has a high degree of credibility to its information source, so it is considered that its degree of freedom is infinite.

3) Uncertainty contribution from test radius error

The measuring radius is determined by a ruler, and its display error is $\pm 1 \mathrm{~mm}$, the influence difference of measuring radius is $\pm 0.014 \mathrm{~dB}(\mathrm{~A})$ calculated from the calculation of relevant items $10 \lg \frac{\mathrm{s}}{\mathrm{S}}$, which is considered to be evenly $\mathbf{S}_{0}$

distributed, so it contains the factor $k=\sqrt{3}$. Thus the uncertainty caused by the measurement radius error is obtained $\boldsymbol{U}_{B 3}=\frac{U}{k}=\frac{0.014}{\sqrt{3}}=0.008 \mathrm{~dB}(A)$, degree of freedom $\boldsymbol{V}_{B 3}=50$

4) Uncertainty contribution from test environment reflection

As the test site is relatively open, the local noise control of the test environment is close to the level of the anechoic chamber, so the impact on the uncertainty is not considered.

5) Uncertainty contribution from temperature, humidity, pressure

During the air noise test of air compressor, the temperature is $34^{\circ} \mathrm{C}$, and the atmosphere pressure is $1000 \mathrm{kPa}$, thus,

$$
K_{5}=\operatorname{lol}\left(\sqrt{\frac{293}{273+\mathrm{t}}} \cdot \frac{P_{0}}{100}\right)=0.010 \mathrm{~dB} \quad \text { (A ). so the }
$$

distribution from temperature, humidity and pressure is $\boldsymbol{U}_{B 5}=K_{5}=0.010 \mathrm{~d} B(A)$, degree of freedom is $\boldsymbol{V}_{B 5}=50$.

According to the formula of A-weighted sound power level noise, the composite uncertainty of this test consists of two parts, they are uncertainty from sound level meter calibration and test radius error. Because the uncertainty components of each standard are not related to each other except the temperature, humidity and atmosphere pressure have negative correlation, meanwhile the measured sound pressure level is positively and negatively correlated with the measured radius. So in summary, the composite uncertainty of this test shall take the maximum value, that is $u_{c}=\sqrt{u_{B 1}^{2}+u_{B 2}^{2}+u_{B 4}^{2}} \pm u_{B 3}-u_{B 5}=0.097 \mathrm{~d} B(\mathrm{~A})$, its degree of freedom is 24 from the calculate formula of composite uncertainty. [6]

The expanded uncertainty is determined as follows:

Confidence level take $\mathrm{p}=95 \%$, degree of freedom take 24 , get $\mathrm{t}=2.09$ from JJF 1059.1-2012, thus the expanded uncertainty $\mathrm{U}=2.09 \times 0.097=0.202 \mathrm{~dB}(\mathrm{~A})$.

\section{Summary}

First of all, from the perspective of scientific measurement and analysis, the test process is strictly carried out in accordance with the standards, and after the test it is essential for testers to summarize and analyze the sources of uncertainty. To evaluate the uncertainty of measurement, first of all, a proper mathematical model should be established according to the function relationship between the measured and its measured composites. The perspective of measuring instrument, measuring environment, personal and measuring methods should be fully considered to make sure the sources of uncertainty, and no omission or repletion shall be made as far as possible, which may cause the final uncertainty deviation. Before calculating the standard uncertainty, we should process the measurement data firstly, minimize the system error, eliminate the gross error and remove the abnormal value, so as to make the uncertainty calculation result more reliable.

Secondly, from the point of view of the steps of air noise measurement method, the uncertainty of measurement results mainly comes from the calibration process, measurement process and equipment accuracy. Therefore, the higher the accuracy of testing and calibration equipment process, and the more conductive to the improvement of measurement accuracy.

Lastly, scientific protection of sound level meter and other precision equipment should be considered especially. The environment in which the sound level meter is placed has a great influence on the sensitivity of the sound level meter, so the effectiveness of measurement can be improved by placing the sound level meter in the incubator after the measurement verification. 


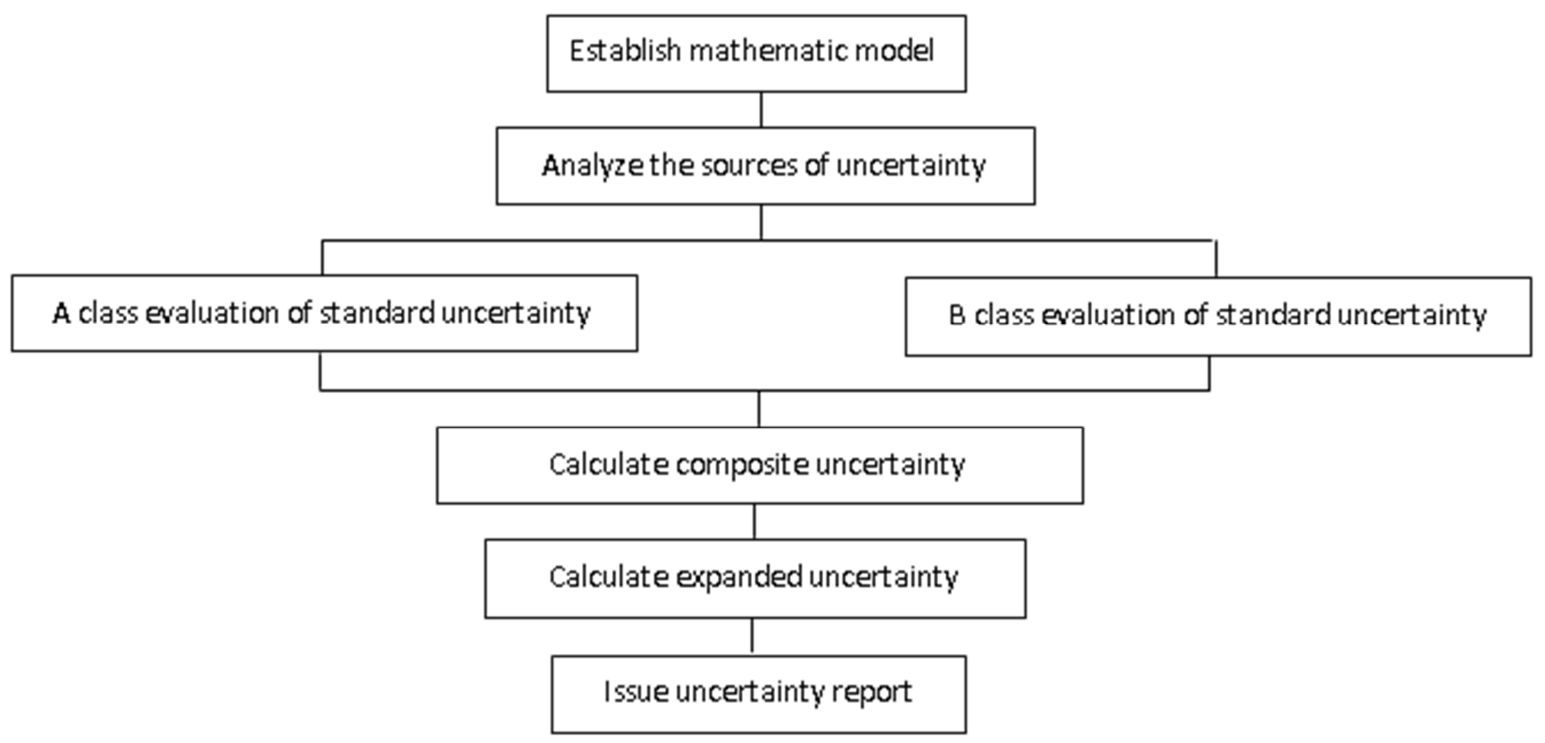

Figure 3. Evaluation process of measurement uncertainty.

\section{References}

[1] Yu Lihua, The importance of measurement uncertainty. [J], Yunnan Electric Power University, 2010, 38 (6): 72-74.

[2] GJB 4058-2000, Measurement method of noise and vibration of ship equipment. [S].

[3] ISO/IEC 17025-2005, General requirements for the competence of testing and calibration laboratories. [S].

[4] JJF 1059.1-2012, Evaluation and expression of measurement uncertainty. [S].

[5] Wang Chengmin, Ma Li, Sun Jia, Evaluation of measurement uncertainty of noise at plant boundary.[J], Environmental protection technology, 2012, 06 (18): 261-262.

[6] $\mathrm{Xu} \mathrm{Bo,} \mathrm{Liu} \mathrm{Congyue,} \mathrm{Uncertainty} \mathrm{analysis} \mathrm{of} \mathrm{phase} \mathrm{noise}$ measurement [J]. Electronic quality, 2008, (01): 9-13.

[7] Fei Yetai, error theory and data processing, Beijing: Machinery Industry Press, 2007.

[8] Ye Depei, measurement uncertainty, Beijing: Bureau of standards and metrology, commission of science, technology and industry for national defense, 1998.

[9] Xue Xiaoyan, interpretation of relevant parameters of air noise sound insulation, acoustic engineering, 2008.
[10] Qiu Lifan, Xi Yinong, Zhou Qingyun. Analysis and evaluation of measurement uncertainty of airborne sound insulation [C] / / Symposium on ship underwater noise for the 30th anniversary of the establishment of the underwater noise group of the academic committee of ship mechanics of China Shipbuilding Engineering Society. 2015.

[11] Cai Yangsheng, Zhao yuezhe. Comparative study on the uncertainty of sound insulation measurement by sound pressure method and sound intensity method $[\mathrm{J}]$. Vibration and shock, 2018, 37 (008): 42-46, 53.

[12] Zhang Rong, Qin Haoming, Li Junzhao. Study on sound insulation test method of wall panel by sound pressure method in reverberation room semi anechoic room [C] 16th academic exchange meeting of China Aviation structure dynamics professional group.

[13] Deng Chunhui, Dong Shuhai, Luo Xizhen. Detection method and system of noise reduction effect of a composite sound insulation material:, cn104749256a [P]. 2015.

[14] Luo Yixi. Development and performance analysis of flexible noise reduction composites [J]. Industrial textiles, 2006 (04): $9-11$.

[15] Luo Yixi, Shaoxing University of Arts and Sciences. Test and analysis of noise reduction performance of flexible noise reduction composites [C] China Textile Industry Association. China Textile Industry Association, 2008. 